\title{
Fungal Flora Contaminating Egyptian Ras Cheese with Reference to Their Toxins and Enzymes
}

\author{
Nermien H. Seddek ${ }^{1}$, Nanis .H.Gomah", ${ }^{2, *}$ Dina. M. Osman ${ }^{2}$ \\ ${ }^{1}$ Botany \&Microbiology Department, Faculty of Science, Assiut University, Egypt \\ ${ }^{2}$ Dairy Science Department, Faculty of Agriculture, Assiut University, Egypt
}

Copyright $\mathrm{O} 2016$ by authors, all rights reserved. Authors agree that this article remains permanently open access under the terms of the Creative Commons Attribution License 4.0 International License

\begin{abstract}
A study was conducted to determine the surface contaminant fungi associated with different samples of Egyptian Ras Cheese collected from different locations in Assiut City, Egypt. 52 fungal isolates assigned to six species belonging to the genera Aspergillus, Penicillium and Mucor were recovered and identified. Aspergillus was the most predominant and represented by four species namely $A$. flavus, A. niger, A. ustus, and A. fumigatus. Penicillium aurantiogriseum and Mucor racemosus were detected as a single representative of the genera Penicillium and Mucor. All the isolated fungal species produced casienase and lipase but did not produce lactase. Analysis of the studied Ras cheese samples for the presence of aflatoxin indicated that $66.6 \%$ of the analyzed samples were contaminated with aflatoxins $\mathrm{B}_{1}, \mathrm{~B}_{2}$ and $\mathrm{G}_{2}$.
\end{abstract}

Keywords Ras Cheese, Aflatoxins, Fungi, Casienase, Lipase, Lactase

\section{Introduction}

Ras (Romy) cheese is the main traditional hard cheese in Egypt, it is manufactured in a high proportion under artisan conditions from raw cow's or mixture of cow's and buffalo's milk without using starter cultures and marketing when it has a queried sharp flavor closed to kefalotyic cheese after 3 to 6 months [Dabiza and El-Deib [1], and Hattem et al. [2]. Generally, Contamination of milk and its products especially ras chees may occur from the raw material or during manufacturing, storage and distribution Kure et al. [3]. . That microorganisms impact the biochemical characters and flavor of such products as well as their appearance rendering them commercially undesirable and often resulting in decreasing the quality of the dairy product Demarigny et al., [4]. and Muir \& Banks, [5].

Contamination of cheeses by filamentous fungi may originate from raw materials such as milk or may be occurred during cheese making either from the environment or are deliberately inoculated using commercial ripening cultures. Delavenne et al., [6] showed that samples of cow milk contained high fungal diversity with up to 15 species in a single sample, whereas a maximum of 4 or 6 different species were recovered in goat and sheep milks, respectively.

Sengun et al. [7] reported that many types of cheese are an excellent substrate for mold growth. Important fungi grown on cheese include Penicillium, Aspergillus, Cladosporium, Geotrichum, Mucor and Trichoderma. These fungi can grow at reduced levels of water activity (aw), and at low $\mathrm{pH}$ value, they also can grow over a wide range of temperature (i.e.0$40{ }^{\circ} \mathrm{C}$ ) and can utilize a wide range of substrates. During their growth on hard cheese, molds are able to produce lipolytic and proteolytic enzymes, which cause deterioration of the product and affect palatability and nutritional value. Consequently, such molds can form gases, rancidity and off flavor due to their lipolytic and protiolytic activity and may produce bitter compounds from lactose as discovered by Viljoen and Greyling [8].

Fungal contamination not only causes deterioration of foods but also adversely affect the human health by production of toxic metabolites called mycotoxins which could be regarded as potential health hazard as Mc Sweeney and Dobson, [9]. Consumption of mycotoxin-contaminated foods has been associated with human mycotoxicosis and sometimes leads to death Bathnagar and Garcia [10]. Mycotoxins can be present in dairy products from two origins: (a) indirect contamination, which results when dairy cows ingest feed that contains mycotoxins that pass into the milk such as aflatoxin M1, and (b) direct contamination, which occurs because of the intentional or accidental growth of molds. They are naturally occurring molecules and are thought to confer a selective advantage to the producer strain within complex ecosystems. Mycotoxin role is to join and compete with other organisms or to inhibit competitor growth and reproduction in the same trophic niche Magan and Aldred [11], Fox and Howlett [12]. Early mycotoxin production could also allow molds to rapidly colonize the environment.

Many food spoilage fungi belonging to the genera 
Aspergillus, Fusarium, and Penicillium produce mycotoxins that are toxic for human and animal. Naturally present in ambient air, soil, and crops Yiannikouris and Jouany [13]., myco-toxigenic fungi are considered to be among the most important contaminants in foods from side of impact on public health, food safety, and the national economy of several countries Steyn [14] and Pitt [15].

Ras cheese is the most preferred and easy food for children and adults in Egypt, especially as a school sandwich or a quick breakfast, so attention to healthy product of Ras cheese is very important for human health. For this reason authors conducted this research. In addition to spread of diseases caused by presence of mycotoxin in food even if with little amount.

There are physical, chemical and biological methods to prevent the fungal growth, eliminate or reduce the toxin levels, degrade or detoxify the toxins in foods and feeds. However, the best way for avoiding the presence of mycotoxins in dairy products is to prevent mold contamination, since there is limitation of degradation or detoxifications the present investigation was designed to study: a) Isolation and identification of spoilage fungal species contaminating different samples of Egyptian Ras cheese; b) Occurrence of mycotoxins in the moldy samples; c) Screening the potentiality of the isolated fungi for mycotoxins and enzymes production.

\section{Materials and methods}

\subsection{Sample Collection:}

Ras (Romy) Cheese samples (three different blocks from different three locations) were collected from different markets of Assiut in 2015. Ten sub-samples were taken from the whole surface of each block and individually kept in sterilized sealed container, transferred to the laboratory and kept in refrigerator to Prepare for fungal survey and mycotoxins analysis.

\subsection{Detection and Isolation of Fungi}

The dilution- plate method of Jarvis et al. [16] was applied for isolation and identification of fungi from the examined cheese samples. Two types of media were used, Potato dextrose agar (PDA) medium by Oxoid [17] and Czapek (CZ) agar medium by Ronald, [18]. Chloramphenicol (20ug/ml) and rose Bengal (30ppm) was used as bacteriostatic agent. Ras cheese wheel surface was scraped and ten gram mixed sample was suspended in $90 \mathrm{ml}$ sterilized distilled water using a rotating shaker to homogenate the obtained suspension. Then, serial ten- fold dilutions were prepared and one $\mathrm{ml}$ of the appropriate dilution was put into a sterilized petri dish then melted medium was poured, mixed well and left to solidify. Ten plates were used for each sample (5 plates for each medium). The plates were incubated at $28 \pm 2^{\circ} \mathrm{C}$ for 5-7 days and the developing colonies were counted and isolated for the identification.

\subsection{Identification of the Isolated Fungi}

Identification of the isolated fungi was carried out on the bases of their macro and microscopic characteristics using the taxonomic methods of Raper and Fennel [19]; Pitt [20]; Moubasher [21]; Ronhede et al. [22] and Rajankar et al. [23]

\subsection{Screening of the Isolated Fungi for Proteolytic and Lipolytic Activity}

Casein hydrolysis by all the isolated fungal strains was estimated using the medium of Ong and Gaucher [24].The clear zone diameter around fungal colony was measured in $\mathrm{mm}$. Lipolytic activity was similarly tested using Czapek agar medium containing fat of milk. Plates were individually inoculated by each fungal strain, incubated at $25^{\circ} \mathrm{C}$ for 7 days. Positive results were recorded after flooding the plate with copper sulfate solution [El-Fadaly et. al [25], Habib, A.A.A. [26].

\subsection{Screening of the Isolated Fungi for Lactase Production}

Lactose fermentation was estimated using Maconkey medium containing $3.0 \mathrm{~g}$ lactose / $\mathrm{L}$ and adjusting to $\mathrm{pH} 0.7$ according the method described by APHA [27].

\subsection{Analysis of Ras Cheese for Mycotoxins Contamination}

Thin-layer chromatography was routinely used for the qualitative analysis of Ras cheese samples for the presence of mycotoxins (if any) according to the method of AOAC [28]. The extraction was performed using chloroform: water $(9: 1 \mathrm{v} / \mathrm{v})$ and the obtained crude extract was purified by column chromatography containing anhydrous sodium sulphate and silica gel. Qualitative estimation was done by comparing of $\mathrm{R}_{\mathrm{f}}$ value fluorescence, color and intensities of the unknown spots with those of the authentic reference of the expected mycotoxins usually produced by the isolated fungi.

\subsection{Quantitative Determination of Aflatoxins by HPLC:}

Detection of aflatoxins concentration in the qualitatively positive samples was performed at the analytical chemistry unit (ACU), Faculty of Science, Assiut University by using of HPLC (Agilent 1200 series, USA). $30 \mu \mathrm{L}$ sample extract was injected in a reversed-phase C18 column (Zorbax, Eclipse $4.6 \times 250 \mathrm{~mm}, 5$-Micron particles), equipped with a security guard cartridge $(2.1 \mathrm{~mm} .12 .5 \mathrm{~mm}, 2 \mu \mathrm{m}$ particles $)$ containing the same stationary phase as the column, at $30^{\circ} \mathrm{C}$. The column was eluted using a gradient flow rate $1.5 \mathrm{~mL} /$ min. of the solvent mixture, water: methanol: acetonitrile $(55: 15: 30 \quad \mathrm{v} / \mathrm{v} / \mathrm{v})$. Post column was UVE LC Tech 
(Photochemical Post column Derivatizer) UVC $254 \mathrm{~nm}$ and Detector was FLD at $295 \mathrm{~nm}$ (excitation) and $330 \mathrm{~nm}$ (emission).

\section{Results and Discussion}

The mycological analysis of the studied Egyptian Ras cheese samples revealed that, a total of 52 and 41 fungal isolates were recovered on PDA and $\mathrm{CZ}$ agar media, respectively (Table1).The isolated fungi were identified as six species belonging to three genera .Taxonomically, the obtained species were assigned to 2 phylla with 2 classes, 2 orders, and 2 families. The family Trichocomaceae (Aspergillus and Penicillium) in the order Eurotiales accommodated the most species (Five species out six), while the family Mucoraceae belonging to order Mucorales represented by one species out of six (Mucor racemosus). Family Trichocomaceae had the highest contribution to the isolated fungi and had high occurrence in three habitats under investigation.

Aspergillus was the most predominant genus encountering in $94.2 \%$ and $90.2 \%$ of the total fungi recovered on PDA and CZ media, respectively. Four species of Aspergilla were identified of which, A. ustus was the most prevalent $(30.77 \%$ and $53.66 \%$ of the total fungi), followed by $A$. fumigatus $(23.08 \%$ and $14.63 \%), A$. flavus $(21.15 \%$ and $12.2 \%$ ) and $A$. niger $(19.23 \%$ and $9.76 \%$ ) of the total fungi isolated on PDA and CZ media, respectively.

Data in Table (1) also showed that Penicillium occupied the second place with regard to the percentage of occurrence. Two isolates of $P$. aurantiogriseum were recovered from one cheese sample representing $3.85 \%$ and $4.88 \%$ of the total fungal count estimated on PDA and $\mathrm{CZ}$ agar media, respectively. Mucor racemosus was detected as a single representative of the genus Mucor. It was isolated only at level of $1.92 \%$ from one sample cultivated on PDA and at level of $4.88 \%$ from two samples cultivated on $\mathrm{CZ}$ agar medium.

The present results are in agreement with those obtained by other investigators. Gandomi et al. [29] reported that, species of Penicilliumand Aspergillus are common contaminants of cheese. Cheong et al. [30] demonstrated that molds such as Aspergillus, Penicillium, Mucor, Cladosporium and Geotrichum are the most common cheese spoilage organisms which can lead to economic loss and raising public health. More recently, El-Fadaly et al. [25] isolated 66 fungal isolates from Ras cheese and classified them into13 species belonging to 6 genera of which, Aspergillus was the most predominant.

Table 1. Total count and frequency of occurrence of fungi isolated from Ras cheese on PDA and CZ agar media using dilution- plate method at three different localities.

\begin{tabular}{|c|c|c|c|c|c|c|c|c|c|c|}
\hline & \multicolumn{9}{|c|}{ PDA } & \multicolumn{5}{c|}{ CZ } \\
\hline & 1 & 2 & 3 & TC & TC\% & 1 & 2 & 3 & TC & TC\% \\
\hline Aspergillus & 14 & 18 & 17 & 49 & 94.23 & 12 & 12 & 13 & 37 & 90.24 \\
\hline A. flavus & 6 & 3 & 2 & 11 & 21.15 & 5 & 0 & 0 & 5 & 12.2 \\
\hline A. fumigatus & 5 & 3 & 4 & 12 & 23.08 & 6 & 0 & 0 & 6 & 14.63 \\
\hline A. niger & 3 & 5 & 2 & 10 & 19.23 & 1 & 2 & 1 & 4 & 9.76 \\
\hline A. ustus & 0 & 7 & 9 & 16 & 30.77 & 0 & 10 & 12 & 22 & 53.66 \\
\hline Penicillium aurantiogriseum & 0 & 2 & 0 & 2 & 3.85 & 0 & 2 & 0 & 2 & 4.88 \\
\hline Mucor racemosus & 0 & 1 & 0 & 1 & 1.92 & 1 & 1 & 0 & 2 & 4.88 \\
\hline Total counts & 14 & 21 & 17 & 52 & 100 & 13 & 15 & 13 & 41 & 100 \\
\hline
\end{tabular}

Table 2. Production of caseinase, lipase and Lactase by fungal species isolated from Ras cheese samples

\begin{tabular}{|c|c|c|c|}
\hline fungi & Lipase & Casinase & Lactase \\
\hline Aspergillus ustus & ++ & + & - \\
\hline Aspergillus flavus & ++ & +++ & - \\
\hline Aspergillus fumigatus & + & + & - \\
\hline Aspergillus niger & +++ & +++ & - \\
\hline Penicillium aurantiogriseum & + & + & - \\
\hline Mucor rasmusus & + & + & - \\
\hline
\end{tabular}

Clear zone diameter $(\mathrm{mm}): \quad+++=>0.2 \quad,++=<0.2, \quad+=>0.1,-=$ no enzyme production 


\subsection{Enzymes Production by the Isolated Fungi}

Results in Table (3) showed that all the isolated fungal species have the ability to produce caseinase and lipase at varying degrees. Aspergillus niger and A. flavus were the highest caseinase producers. In contrarily, A. ustus and $A$. fumigatus showed the lowest ability of casienase production. At the same time, Penicillium aurantiogriseum exhibited moderate degree of casein hydrolysis. Data in Table (3) also revealed that $A$. niger was the highest powerful in lipase production, followed by $A$. ustus and $A$. flavus. Meanwhile $A$. fumigatus, Mucor rasmusus and Penicillium aurantiogriseum secreted the lowest lipolytic activity. The obtained results are in agreement with that reported by Ismail [31], who isolated 108 fungal strains belonging to 31 genera from the Egyptian foodstuff Kishk and found that all the isolated strains are capable of producing casienase and catalase.

On the other hand, all the examined fungi were unable to produce lactase. However, lactose assimilation might be not important to affect growth of contaminant fungi because of the presence of starter cultures which can produce lactase.

The obtained results can give an idea about the degradation processes and flavor developing in Ras cheese in the presence of fungal contamination.

Mc Sweeney and Sousa [9] demonstrated that most lactose in milk is lost in whey as lactose or lactate during cheese manufacture. However, low levels of lactose remain in the crude at the end of manufacturing $(0.8 \%-1.0 \%)$. Residual lactose is metabolized quickly to lactate (Glycolysis) during the early stages of ripening at a rate largely determined by temperature and the salt- moisture(S/M) levels of the crud.

Table 3. Aflatoxins contamination levels detected in some samples of Egyptian Ras cheese

\begin{tabular}{|c|c|c|}
\hline Samples & Type of aflatoxin & Conc. $\mu \mathrm{g} / \mathrm{kg}$ \\
\hline \multirow{3}{*}{ No.1 } & $\mathrm{B} 1$ & 2.62 \\
\cline { 2 - 3 } & $\mathrm{B} 2$ & 1.48 \\
\cline { 2 - 3 } & $\mathrm{G} 2$ & 0.69 \\
\hline \multirow{3}{*}{ No.2 } & $\mathrm{B} 1$ & 6.31 \\
\cline { 2 - 3 } & $\mathrm{B} 2$ & 3.14 \\
\cline { 2 - 3 } & $\mathrm{G} 2$ & 0.87 \\
\cline { 2 - 3 } No. 3 & $\mathrm{~B} 1$ & N. D. \\
\cline { 2 - 3 } & $\mathrm{B} 2$ & N. D. \\
\cline { 2 - 3 } & $\mathrm{G} 2$ & N. D. \\
\hline
\end{tabular}

\subsection{Natural Occurrence of Aflatoxins in Ras Cheese}

AS shown from data presented in Table (1), Aspergillus flavus (known as a potent aflatoxin- producing fungus) was isolated from all the studied Ras cheese samples. This observation raising the possibility for contaminate of cheese with aflatoxin. Therefore, the most contaminated sample from each location was analyzed for the presence of aflatoxins. Data in Table (3) showed that two samples out of the three analyzed were contaminated with aflatoxins $B_{1}, B_{2}$ and $\mathrm{G}_{2}$ at concentrations ranged between $2.61-6.31,1.48-$ 3.14 and $0.69-0.87 \mu \mathrm{g} / \mathrm{kg}$, respectively. The third analyzed sample proved to be aflatoxin-free although it was contaminated with Aspergillus flavus. In this respect, Drusch and Aumann [32] demonstrated that Mycotoxins can diffuse into the environment and can be found in food or feed areas, which do not show any sign of mycelium growth. Therefore, the absence of molds does not guarantee freedom from mycotoxins, and conversely, the presence of a toxinproducing mold does not automatically imply the presence of mycotoxins in food and feed.

The obtained results are in agreement with that reported by other investigators as Siemens and Zawistowski, [33] who's found the incidence of mycotoxins at different concentrations in various types of cheese. Lieu and Bullerman [34] showed that aflatoxinB1 and G1 were stable in cheese during storage at $5^{\circ} \mathrm{C}$. Sengun et al. [7] reported that cheese is a very susceptible product for mold growth and also production of mycotoxins. Although there are some methods to control or detoxify the mycotoxins, the effects of these methods are limited and also have some restrictions in the application. So, the best way for avoiding mycotoxins in cheese is to prevent mold growth.

\section{Conclusions}

All the studied Ras cheese samples were contaminated with different species of molds. Most fungal isolates were able to secrete proteolytic and lipolytic enzymes, which may cause deterioration of the cheese and alter its palatability and nutritional value. Also some molds have the ability to produce mycotoxins which are harmful for consumers. Therefore, car should be taken to prevent mold growth on cheese surface during ripening, storage and handling.

\section{REFERENCES}

[1] Dabiza, N., and El-Deib, K., Biochemical evaluation and microbial quality of Ras cheese supplemented with probiotic strains., Polish J. food and nutrition sciences, 2007 ; 57(3), 255-300.

[2] Hattem, H. E, Taleb, A. T., Manal, A. N., and Hanaa, S. S., Effect of pasteurization and season on milk composition and ripening of Rascheese., J. Brewing and Distilling, 2012 ; 3(2), 15-22.

[3] Kure, C.F., Skaar, I and Brendehaug, J. 2004. Mould contamination in production of semi-hard cheese. Int. J. Food Microbiology. 15:93:41-49.

[4] Demarigny, Y., Beuvier, E., Buchin, S., Pochets, S. and Grappin, R. 1997. Influence of raw milk microflora on the character of Swiss type cheese. Biochemical and sensory characteristics Lait., 77:151-167.

[5] Muir, D.D. and Banks, J.M. 2000. Milk and Milk products in the stability and shelf life of food. BocaRaton. FL. 197- 219.

[6] Delavenne E, Mounier J, Asmani K, jany J-L, Barbier G, Le 
Blay G. 2011. Fungal diversity in cow, goat and ewe milk. Intl J Food Microbiol 151:247-51.

[7] Sengun I.Y., D.B. Yaman and S.A. Gonul Mycotoxins and mould contamination in cheese: a review World Mycotoxin Journal, August 2008; 1(3): 291-298

[8] Viljoen, B.C., Greyling, T.,. Yeasts associated with Cheddar and Gouda making. Int. J. Food Microbiol. 1995; 28, 79-88.

[9] Mc Sweeney P L H and Sousa M J Biochemical path-ways for the production of flavour compounds in cheese during ripening. Le Lait, 2000; 80293 - 324.

[10] Bathnagar, D. and Garcia, S. A. Guide to Food borne Pathogens. John Wiley and Sons Publish, 2001; pp. 35-49.

[11] Magan N, Aldred D. 2007. Why do fungi produce mycotoxins? In: Dijkserhuis J, Samson RA, editors. Food mycology: a multifaceted approach to fungi and food. New York: CRC Press/Taylor and Francis Group. P. 121-33.

[12] Fox EM and Howlett BJ. 2008. Secondary metabolism: regulation and role in fungal biology. Curr Opin Microbiol 11:481-7.

[13] Yiannikouris A, Jouany JP. 2002. Mycotoxins in feeds and their fate in animals: a review. Anim Res 51:81-99.

[14] Steyn PS. 1995. Mycotoxins, general view, chemistry and structure. Toxicol Lett 82(8):843-51.

[15] Pitt JI. 2000. Toxigenic fungi and mycotoxins. Br Med Bull 56:184-92.

[16] Jarvis, B.; Seiler, D. A.; Ould, A. J. and Williams, A. P. Observations on the enumeration of moulds in food and feeding stuffs. Journal of Applied Bacteriology, 1985; 55, 325-336.

[17] Oxoid, The Oxoid Manual. 9th ed., by OXOID Limited, Wade Road, Basingstoke, Hampshire RG24 8PW, England, UK, 2006.

[18] Ronald, M. A., Hand Book of Microbilogical Media for the Examination of Food. CRC Taylor and Francis Group Boca Raton London New York, 2006.

[19] Raper KB \& Fennell DI (1965). The Genus Aspergillus, $p p$ 686 Baltimore: Williams \& Wilkins Company.

[20] Pitt J.I. The genus Penicillium and its teleomorphic states Eupenicillium and Talaromyces Academic Press Inc, London, 1979.

[21] Moubasher, A. H. Soil Fungi of Qatar and other Arab Countries- The Scientific and Applied Research Centre,
University of Qatar, 1993; 566 pp.

[22] Ronhede, S.; Jenesen, B.; Rosendahl, S.; Kragelund, B.B.; Juhler, R.K. and Aamand, J. Hydroxylation of the herbicide isoproturon by fungi isolated from agricultural soil. Applied and Environmental Microbiology, 2005; 71(12): 7927-7932.

[23] Rajankar, P.N.; Tambekar, D.H. and Wate, S.R. Study of phosphate solubilization efficiencies of fungi and bacteria isolated from saline belt of purna river basin. Research Journal of Agriculture and Biological Science, 2007; 3(6):701-703.

[24] Ong PS, Gaucher GM. Protease production by thermophilic fungi. Can. J. Microb., 1973; 19: 129 - 133.

[25] El-Fadaly, H., El-Kadi, S.M., Hamad, M.N.F. and Habib, A. Isolation and Identification of Egyptian Ras Cheese (Romy) Contaminating Fungi during Ripening Period. Journal of Microbiology Research, 2015; 5, 1-10.

[26] Habib, A.A.A. Studies on Fungal Decontamination of Ras Cheese during Storage by Using Some Natural Substances .Master's Thesis, Microbiology Department, Faculty of Agriculture, Damietta University, Damietta, 2014.

[27] APHA, American Public Health Association Standard Methods for the Examination of Water and Wastewater.20th Edition, APHA, Inc., New York, 1998.

[28] Official Analytical Chemists (AOAC), 1984.

[29] Gandomi H, Misaghi A, Basti AA, Bokaei S, Khosravi A, Abbasifar A, Javan AJ Effect of Zatariamultifora Bioss. Essential oil on growth and aflatoxin formation by Aspergillus flavusin culture media and cheese. Food and Chemical Toxicology, 2009; 47: 2397-2400.

[30] Cheong YL, Burkart K, Leitão PJ, Lakes T. Assessing weather effects on dengue disease in Malaysia. Int J Environ Res Public Health, 2013; 10(12):6319-34.

[31] Ismail, M. A. Degradative enzymes and fungal flora associated with the Egyptian foodstuff Kishk. International Biodeterioration \& Biodegradation, 1993; 31: 143-157.

[32] Drusch and Aumann Mycotoxins in fruits: Microbiology, occurrence and changes during fruit processing. Advances in Food and Nutrition Research, 2005; 50: 33-78.

[33] Siemens, K., Zawistowski, J., Occurrence of PR imine, a metabolite of Penicillium roqueforti, in blue cheese. Journal of Food Protection, 1993; 56 (4), 317-325.

[34] Lieu, F.Y. and L.B. Bullerman. Production and Stability of Aflatoxin, Penicillic Acid and Patulin in Several Substrates. Jour. Food Science, 1977; 42:1222-1224, 1228. 